\title{
A century of bias in genetics and evolution
}

\author{
Laurence D. Hurst ${ }^{1}$
}

Received: 8 November 2018 / Revised: 29 January 2019 / Accepted: 29 January 2019

(c) The Genetics Society 2019

\begin{abstract}
Mendel proposed that the heritable material is particulate and that transmission of alleles is unbiased. An assumption of unbiased transmission was necessary to show how variation can be preserved in the absence of selection, so overturning an early objection to Darwinism. In the second half of the twentieth century, it was widely recognised that even strongly deleterious alleles can invade if they have strongly biased transmission (i.e. strong segregation distortion). The spread of alleles with distorted segregation can explain many curiosities. More recently, the selectionist-neutralist duopoly was broken by the realisation that biased gene conversion can explain phenomena such as mammalian isochore structures. An initial focus on unbiased transmission in 1919, has thus given way to an interest in biased transmission in 2019. A focus on very weak bias is now possible owing to technological advances, although technical biases may put a limit on resolving power. To understand the relevance of weak bias we could profit from having the concept of the effectively Mendelian allele, a companion to the effectively neutral allele. Understanding the implications of unbiased and biased transmission may, I suggest, be a good way to teach evolution so as to avoid psychological biases.
\end{abstract}

\section{Introduction}

I am somewhat reluctant to admit that when I first met genetics as a subject I was, shall we say, less than enthusiastic. Some monk far away (in time and space) bred some peas and he found that heterozygotes mated to heterozygotes leave three of one sort to one of the other. How interesting (I did not think). In part, my lack of enthusiasm was understandable. I knew about meiosis-and had been made to learn the names of all the stages-and when you know about mutations, DNA, chromosomes and meiosis, which of course Mendel did not know, then 3:1 ratios are simply an obvious consequence (assuming you add in a hefty dose of dominance). I similarly could not see the excitement in a Punnett square-it just seemed too simple.

The seemingly trivial result from Mendel, however, presents a circumstance possibly unique in science, one in which a central theory requires a particular parameter to be a very particular value. The parameter in question is the extent of deviation alleles receive in meiosis away from

Laurence D. Hurst

1.d.hurst@bath.ac.uk

1 The Milner Centre for Evolution, University of Bath, Bath BA2 7AY, UK
50:50 segregation in heterozygotes. In this opinion piece, I note that, both at the start of the history of The Genetics Society and now, this parameter was and is of great importance. A century ago, it was important because the realisation that inheritance is particulate and meiosis is typically "fair" (i.e. no deviation from 50:50) has important theoretical consequences for the preservation of variation and, in turn, the role of selection. Now it is of interest because it seems clear that, at least in some genomes, there are regular deviations from 50:50 and that this nonMendelian transmission probably resolves recent debates between selectionists and neutralists (e.g. the isochore debate) in favour of neither. It may even explain, at least in some instances, why speciation happens. However, our abilities to resolve subtle but potentially important biases are currently facing technical limitations owing to methodological biases. More generally, the relationship between transmission bias (or lack thereof) and our understanding of evolution, highlights the importance of having a strong rooting of evolutionary genetics in molecular and transmission genetics: now more than ever, we need all branches of genetics to be integrated under one umbrella. The same intimate link between genetics and evolution is for the most part ignored in UK school teaching. Understanding of the role of bias in transmission is, I suggest, a helpful way of explaining the relationship between the two, so overcoming 
psychological biases that can be an impediment to learning owing to cognitive dissonance (Festinger 1957).

\section{Fitness isn't so important if inheritance isn't Mendelian}

Mendel's major insight was that inheritance is particulate. Heterozygotes have two different versions of these particles - he did not call them alleles, but would do so now. Let us ask about the population genetics of an imaginary sexual species that is randomly mating just by considering what the fate of these alleles will be. I'll suppose there are only two such alleles and neither confers a fitness advantage over the other. I'll need to know something else: the rate at which my two particles are transmitted from heterozygotes to the next generation. Let me suppose that for one of them this rate is $0.5+k$ and so for the other the rate is $0.5-k$ (with $k \geq 0$ ). Now let me ask about what is going to happen as I forward project in time so as to determine what their longerterm fates will be. The oddity is that unless $k=0$, one of the two alleles will go to fixation because of the transmission bias: the allele with transmission rate $0.5+k$ is the winner in this game.

This is a mathematically simple result. Neither allele affects your fitness but owing to segregation distortion, i.e. by being transmitted at greater than Mendelian rates $(k>0)$, one allele will spread through the population regardless. Although mathematically simple, this is also a profound result. That Punnett square and those 3:1 ratios, all assume not simply particulate inheritance, but that $k=0$ so that alleles are transmitted at equal rates through meiosis. The Punnett square works because each box in the square has the same probability. Importantly, when we add in fitness into the equation, only when $k=0$ do we find that the only parameter that matters is the effect on fitness. If this isn't true, natural selection operating on organismal fitness would be just one player in the game. Indeed, alleles that are deleterious can spread so long as $k>0$ and the larger the value of $k$, the more deleterious the allele can be (Bruck 1957; Hiraizumi et al. 1960; Prout 1953; Sandler and Novitski 1957). If $k>0$, we would not be talking about survival of the fittest (which focuses on phenotypic effects of alleles), but instead on persistence of the allele with the ability to transmit at high rates through meiosis.

\section{The $k>0$ problem and Jenkin's swamping arguments are similar}

The problem of the impotence of natural selection when $k>$ 0 is not greatly dissimilar to the problem raised by the electrical engineer Fleeming Jenkin (Jenkin 1867) in objection to the notion of natural selection. Jenkin thought inheritance was blending rather than particulate, but the core problem is the same. Jenkin's swamping argument, as usually presented (for critique see Morris 1994), was that if inheritance was blending, then new advantageous traits would have difficulty in spreading by selection, as selection had to fight against the mode of inheritance and variation will be lost. Jenkin considers the case of a shipwrecked white man on an island of (what he assumed to be inferior) "negroes". He presumes that "our shipwrecked hero would probably become king; he would kill a great many blacks in the struggle for existence; he would have a great many wives and children, while many of his subjects would live and die as bachelors... In the first generation there will be some dozens of intelligent young mulattoes, much superior in average intelligence to the negroes. We might expect the throne for some generations to be occupied by a more or less yellow king; but can anyone believe that the whole island will gradually acquire a white, or even a yellow population?" (Jenkin 1867). Jenkin's biases here are selfevident. Moreover, ironically, Jenkin did in fact make a miscalculation in a numerical example he presented (Davis 1871) (for discussion see Bulmer 2004). Nonetheless, the point remains that selection can have a problem if it must fight against the mode of inheritance and, unless there is a high mutation rate, variation will tend to be lost.

It was only a decade before the founding of The Genetics Society, that Hardy (1908) and Weinberg (1908) derived their striking result that under unbiased Mendelian inheritance variation was preserved and, so the story goes, evolution by natural selection was rescued as a concept (Morris 1994). But, this intellectual rescue requires that our transmission parameter $k$, take one value, i.e. zero. Normally in science this would be regarded as special pleading. Typically, if you construct a mathematical model, you look to see how broad the parameter space is for your model to work. If this space is small, then your model is looking a bit flimsy. If it requires one value alone, it is usually dismissed out of hand: in an infinitely large world of possible parameter values, to demand that one value alone is required is a pretty remarkable requirement. Indeed, I know of no other model in science of any flavour where such an important body of theory starts by presuming the one value of a parameter necessary for the models to work. But this is what the vast bulk of population genetics does and is the cornerstone of why we think selection (organismic fitness) is so important.

Above I suggested that if $k$ is not zero, we would not be talking about survival of the fittest. I'm not sure we would be talking at all. Could a complex meiotic species viably exist if segregation is consistently non-Mendelian? It is quite hard to see how any meiotic species could adapt to changing conditions if the primary determinant of the fate of 
an allele is not its effect on fitness but is instead its transmission rate through meiosis. You might imagine that such species would adapt by preventing heterozygosis-associated transmission (e.g. by being asexual, by inbreeding or by forcing uniparental inheritance). But several problems present themselves. First could a species with non-Mendelian inheritance at all loci evolve modifiers to control nonMendelian inheritance? There is a large literature on modifiers to control the way genes are inherited (Feldman and Otto 1991), but they start by presuming that the modifier is itself Mendelian. As a philosophical enterprise it would be intriguing to see how the world might look differently. The second problem, is that even if selection of some form could favour asexuality/selfing/uniparental inheritance then the lineages become actually or effectively asexual. But asexual multicellular lineages tend to go extinct and non-meiotic lineages tend not to be multicellular. Prokaryotes (eubacteria and archaea) have, for example, been around for several billion years but never evolved complex multicellularity (for discussion as to why see Lane 2014; Lane and Martin 2010, 2016; Lynch and Marinov 2016, 2017, 2018).

\section{Why might $k=0$ ?}

The argument that, for evolution by natural selection to be a common and viable mode of evolution $k=0$ is necessary, might be misinterpreted as meaning that evolution by natural selection is somehow unlikely. Before anyone suggests that I am advocating some strange argument for the existence of a parameter-fixing god, I am not. That $k=0$ is not some miracle. It follows as the default value if nothing is happening except for meiosis. We do not need special pleading to address this problem. Put differently, that the chromosomal theory of inheritance (Boveri 1904; Sutton 1902, 1903) so neatly explained Mendelian ratios (Carothers 1913), not only explains why the chromosomal theory came to be accepted (Crow and Crow 2002), but also, in turn, provides a defence for considering Mendelian ratios as the default.

This being said, others have considered the problem of how $k=0$ might evolve, were $k=0$ not the default (see, e.g. Crow 1991; Eshel 1985). Leigh conjectures about a parliament of the genes where majority opinion has sway (Leigh 1971). While such language may be attractive, what this means in practice is less then transparent. Consider, for example, modifiers that suppress segregation distorters. The difficulties here are multiple. First, if the suppressor is costly, a balance between the persistence of the distorting allele and the suppressor can be found (unpublished), so Mendelian segregation need not be the equilibrium solution. Second, such models presume nearly all genes, the suppressive modifiers included, are Mendelian and the locus with $k>0$ is the exception. What would happen in a system in which non-Mendelian inheritance was the default is to the best of my knowledge unknown. Third, such an argument may be unnecessary. Autosomal alleles with $k>0$ can rapidly spread to fixation if the distorter homozygotes aren't of too low fitness. At fixation, as everyone has the distorting allele, there is no distortion (at least at that locus for the time being). If the distorter has a high value of $k$ and was sexlinked the population could go extinct owing to a dearth of one of the two sexes (such an X v Y or Y v X distorter will result in heavily biased sex ratios). Either way, the extant populations have no distortion.

Thus, while $k=0$ looks like a fine democratic outcome, it could be either the default or the consequence of an inability of any system to do much about rapid spread. Indeed, in fungi, the species with segregation distorters tend to be more inbred ones (van der Gaag et al. 2000). Inbreeding slows the transit of distorters making it more likely that we will observe them as they slowly proceed to fixation (van der Gaag et al. 2000). We can then extrapolate to suppose that absence of distortion in outbred fungi is a consequence of rapid spread to fixation of such distorters rather than their absence. Similarly, the well described autosomal distorters ( $S D$ in flies, $t$-complex in mice) tend to be homozygous steriles or lethals, either owing an intrinsic consequence of the mechanism of action or owing to linked alleles. This low homozygous fitness prevents their fixation and thus makes it easier for us to discover them. How often then are populations affected by very rapidly spreading segregation distorters with high homozygous fitness? In addition, these and other well described meiotic drive genes are two tightly linked loci (toxin and antidote)(Lyttle 1991) and so are probably hard to mutationally assemble. This being said, there are much simpler modes of transmission distortion (e.g. GC-biased gene conversion, of which more later). Nonetheless, I'm not convinced we need a special case to explain why $k=0$.

\section{The slow rise of non-Mendelian genetics}

If in the early part of the twentieth century deviations from Mendelian inheritance were not given much prominence, it is most likely because most instances of classical single locus conditions are Mendelian (or at the very least, very hard to distinguish from Mendelian). The rise of the chromosomal theory of inheritance (Boveri 1904; Sutton 1902, 1903) and with it the logic of Mendelian segregation ratios (Carothers 1913), no doubt also played a role in seeing Mendelian inheritance, quite correctly, as the default mode of inheritance. Nonetheless, deviations from classical Mendelian inheritance did crop up in the early decades of 
the last century. Mice homozygous for the Yellow allele, for example, are never witnessed in heterozygote $\times$ heterozygote crosses (Castle and Little 1910). While sometimes appearing in textbooks as exceptions or extensions to Mendelian inheritance, these could just as well be viewed as Mendelian with respect to transmission but with early viability selection. Indeed, had this been post-natal inviability, this is probably how they would be classified. Similarly, uniparental cytoplasmic inheritance was discovered early (this is usually jointly credited to Correns and Baur, but Baur seems to have been clearer on the subject (Hagemann 2000)). From a population genetics point of view uniparental inheritance, although non-Mendelian, is not the same as biased transmission, as on the average any given allele is transmitted $50 \%$ of the time-it just happens to be $100 \%$ from one sex and $0 \%$ from the other, rather than $50 \%$ from each. Similarly, unbiased gene conversion is unlikely to have a deterministic effect: for every case that a locus is subject to 3:1 gene conversion in a tetrad there can be an equal but opposite 1:3 event (Gutz and Leslie 1976). None of these phenomena thus disturb the notion of unbiased transmission.

The earliest consideration of the possibility of biased transmission that I am aware of is pre-Mendelian. Darwin thought, at one point, that gemmules moved from the body to the gonads and these formed the basis of inheritance (Darwin 1868) (he often changed his mind about inheritance, possibly owing to Jenkin's objection). He considers the problem of biased transmission of such gemmules as possibly explaining increases in the numbers of body appendages. He conjectures "As the cells of adjoining or homologous parts will have nearly the same nature, they will be liable to acquire by variation each other's elective affinities; and we can thus to a certain extent understand such cases as a crowd of horns on the heads in certain sheep, of several spurs on the leg, and of hackles on the head of the fowl" (p. 393). However, as this was part of a longer narrative based on the incorrect notion of pangenesis, it can hardly be counted a major contribution to the field, although it does highlight that thinking about bias in transmission is not new.

In the early post-Mendelian era (i.e. after circa 1900-1930) there were several parallel discoveries of deterministically biased transmission (e.g. Correns 1902; Gershenson 1928; Heribert-Nilsson 1920; Metz and Moses 1923; Morgan et al. 1925), including sex ratio distorters (Simmonds 1923a, b, 1926, 1928) with one such observation possibly as early as 1911 (Federley 1911, 1936). For consideration of Renner's extensive work on nonMendelian inheritance in Oenothera, see Sturtevant (1926). While the genetics is often well described, consideration of the population genetical impact of such biased transmission was, however, often a little confused.
Consider, for example, Gershenson's analysis of an Xlinked meiotic drive gene causing all female progeny in Drosophila (Gershenson 1928). He argues that "since it exists in the natural population it is probably useful, or at least harmless for the evolution of the given Species". This is the usual backwards Darwinian logic-if it is observed it must be beneficial. Strikingly he then also notices that "the extension of this gene among the wild population must be expected even without the action of positive selection. Usually a sex-linked gene is transmitted by the father to a half of the descendants only, whereas here all or nearly all the flies receive it with the X-chromosome of the father; this favors its extension". Although confused, this does seem to be the first understanding that alleles can deterministically spread owing to biased transmission.

If Gershenson was simultaneously confused but also perspicacious enough in realising that biased transmission alone could lead to invasion, Haldane (1932) a few years later was characteristically clearer, but dismissive of the potential importance of biased transmission. He considered certation, the biased transmission of certain alleles of a heterozygote through pollen (Heribert-Nilsson 1920, 1923). $\mathrm{He}$ comments that because of such a bias, a plant is "at the mercy of its pollen grains", noting that an allele could spread even if mildly deleterious or unable to spread even if advantageous to the plant, if it was uncompetitive in pollen. Curiously, however, while relating that he once considered the phenomenon to be of "overwhelming importance", he concludes by dismissing it, arguing that selective intensity increases only very slowly with intensity of competition (see also Leigh's afterword to the 1990 edition of The Causes of Evolution (Leigh 1990)). For animals, he considers the phenomenon irrelevant as sperm do not express their haploid genotype. One wonders if his view would have changed were he made aware of Gershenson's work.

While the idea that biased transmission enables alleles to invade a population even if deleterious to the bearers of such alleles appears not to have gained great traction after Haldane's considerations, like all good ideas it resurfaced independently (or so I presume) over a decade later. Östergren (1945) argued that non-Mendelian B chromosomes could be genomic parasites. Noticing their ability to be present in more progeny than they would be were they to segregate in Mendelian ratios he argues that "it is obvious that they would get a considerable spread even if their effect on the plants was quite unfavourable", concluding that "they need not be useful to the plants, they need only be useful to themselves". Here he was arguing against Cyril Darlington who took the standard Darwinian line that B chromosomes must have a utility (i.e. increase organismic fitness) (Darlington and Thomas 1941), conjecturing roles in nucleic acid metabolism. In this context, striking contrast can also be made with Östergren on the one hand, and 
Lewis (1941) who just a four years earlier had mathematically modelled the logically comparable phenomenon of cytoplasmic male sterility (CMS). The mitochondrial mutations that induce CMS are also one class of selfish genetic element, the general term for alleles that invade owing to transmission bias (of any flavour) while potentially being deleterious. In CMS the mutation sterilises male tissue, so distorting the hermaphroditic plant's reproductive investment towards egg production away from pollen production. As mitochondria are maternally transmitted, this enables the mutation to spread in the population, even though it is deleterious, but also creates the conditions for the spread of suppressor mutations. While Östergren's arguments were all verbal, Lewis (1941) lays out the maths of CMS, notes how broad the conditions for spread are, but simultaneously conjectures what the advantage must be to the plant to have such alleles (he conjectures a reduction in selfing). He does not notice that the allele could be deleterious and still spread, even though it is implicit in the maths.

While Östergren is sometimes thus credited as one of the first to provide a clear argument to the effect that alleles can be deleterious and still spread if they have a transmission advantage, the importance of the paper may be more profound in retrospect than it was at the time. According to the Web of Science, the paper has been cited only a little over 100 times, initially only within the B chromosome literature. Indeed, it was not until 1980, when Cavalier-Smith drew the attention of the proponents of the selfish DNA hypothesis to the fact that they should have acknowledged Östergren (Cavalier-Smith 1980), that it makes its way out of the more specialist literature. Evidence would suggest that instead the discussion of Sandler and Novitski (1957), a further decade on from Östergren, is perhaps the first to provide an influential assessment of the evolutionary implications of the problem of biased transmission (and coined the term meiotic drive). This has been cited over 350 times. They rely on the rarely cited mathematical model by Prout, an appendix to the rarely cited Dunn (1953). For fuller mathematical treatment, see Hiraizumi et al. (1960). In part, the difference in impact may lie in the accessibility of the journals concerned (American Naturalist as opposed to Botaniska Notiser) rather than any important difference in what was being argued, a common cause of bias in the reporting of the scientific literature.

There is a history to be written about the rise of the understanding that alleles can spread and be deleterious, about the importance (or lack thereof) of Haldane's dismissal and the influence (or lack thereof) of Östergren's insights. Central to any such history would be Hamilton's insights and the refocusing of the conceptualisation of evolution at the allele level (popularised a decade later by Dawkins). Hamilton in his sex ratio paper (Hamilton 1967), for example, is transparent that sex ratio distorting chromosomes ( $\mathrm{X} \vee \mathrm{Y}$ or $\mathrm{Y} \vee \mathrm{X}$ distorters) can invade and either potentially send a population extinct or provide the conditions favouring alleles that suppress the distortion.

Core to the new view of alleles with biased transmission is that, while they can both spread and be deleterious, they can also thus create conditions for suppressors (Hiraizumi et al. 1960; Östergren 1945), hence cause genetic conflicts (Burt and Trivers 2008; Cosmides and Tooby 1981; Hurst et al. 1996; Östergren 1945; Werren 1987). Here again, Östergren (1945) was clear arguing that we expect plants to evolve suppressors of B chromosomes and for the B chromosomes in turn to counter-adapt. He argued that "If the fragments are unfavourable to the plants, there should tend to accumulate in the population factors of a type inhibiting their continued spread" and that "This tendency to the evolution of an »eliminative system» in the normal complement would be counteracted by a tendency to the evolution of an »accumulative system» in the fragment itself. Selection would favour fragments with a more efficient spreading mechanism". Haldane's understanding that advantageous alleles cannot invade if they suffer biased transmission that acts against them, suggests that he would easily have understood the same concepts, he just did not pick up the ball and run with it, so to speak.

Selfish elements and the response they provoke can help explain all sorts of apparently strange phenomena, although historically conjecture has been more prominent than evidence, although that is changing. In particular, the notion that selfish elements spread because they can be deleterious, makes them attractive candidates for mediators of hybrid disruption (Frank 1991; Hurst and Schilthuizen 1998; Hurst and Pomiankowski 1991), for which striking evidence now exists (Hauschteck-Jungen 1990; Phadnis and Orr 2009; Tao et al. 2001) (see for reviews see (McDermott and Noor 2010; Patten 2018; Presgraves 2010)). As there are multiple reviews on selfish elements and genetic conflicts (Agren and Clark 2018; Burt and Trivers 2008; Hurst et al. 1996; Werren 2011; Werren et al. 1988), I'll not indulge at length save to provide one exemplar, the strange case of alleles that select for ever earlier death of embryos.

At first sight, selection should never favour alleles that kill the embryos they are in. But selfish elements are odd. The $t$-complex in mice is a meiotic drive gene complex on chromosome 17. It appears that an intrinsic component of the way drive acts is that in homozygous form it causes males to be sterile (for discussion see Lyon 1992, 2003). The drive chromosome is held in the population by a balance of drive in heterozygotes increasing its frequency and homozygous sterility preventing it from going to fixation. The species in question is a mouse. Mice have continual supply of resources as embryos. But what is the point in investing in an embryo that will grow up to be infertile? If 
these sterile progeny could be killed in utero, resources could then be freed up for other progeny. Thus we have the strange circumstance of the evolution of embryonic mortality in which mutations act to kill early rather than to prevent such mortality (Charlesworth 1994a). As expected, the lethal alleles are in linkage disequilibrium with the $t$ complex and are recessive (Charlesworth 1994a).

\section{Can $k>0$ explain isochores?}

Examples usually considered in the context of selfish elements have very strong distortion phenotypes: nearly all the offspring in Gershenson's X-linked drive example were male and nearly all progeny of male heterozygotes for $t$ complex or $S D$ inherit the distorter. Less experimental effort has been given to weak distorters, in no small part because these are harder to detect. Recent technological innovations, notably whole genome sequencing permitting affordable parent-offspring sequencing, is now changing matters. Here I am thinking in particular of biased gene conversion (BGC) (Brown and Jiricny 1988; Lamb 1984, 1985) (for reviews see Duret and Galtier (2009) and Marais (2003)).

In meiosis, when one strand invades another from an homologous chromosome a heteroduplex is formed. This can be associated with crossing over or non-crossover recombination. This heteroduplex is unusual double stranded DNA as there will be mismatches at any sites that are different between the two homologues. The question is what to do with the mismatches. Let's suppose we have a T:C mismatch. One possibility is to toss an unbiased coin to decide whether to replace the $\mathrm{C}$ with an $\mathrm{A}$ or the $\mathrm{T}$ with a $\mathrm{G}$. Both will give a gene conversion event, but there will on the average be no bias to this process. We will see a 3:1 segregation in any given tetrad, but at the population level, with the $\mathrm{T}->\mathrm{C}$ rate the same as $\mathrm{C}->\mathrm{T}$ rate, there is no effect on allele frequencies. But what if the process is biased? Bengtsson (1985) has suggested there may be good reason for it to evolve to be biased. Mutation is more commonly in the direction GC->AT, so at a T:C mismatch, it is more likely that $\mathrm{C}$ is the ancestral allele and $\mathrm{T}$ the more recent mutant. As most mutation is deleterious, evolution should favour the $\mathrm{C}$ over the $\mathrm{T}$ at the mismatch, thereby correcting a mutation. The best evidence we have from mammals and birds is that, indeed, gene conversion can be biased in favour of GC alleles over orthologous AT alleles. Such a bias is seen in humans (68\% AT->GC) (Halldorsson et al. 2016; Lesecque et al. 2013; Williams et al. 2015) and flycatchers (59\% AT->GC) (Smeds et al. 2016). BGC is also present in honey bee (Wallberg et al. 2015) but possibly absent in Drosophila (Robinson et al. 2014). Note that the numbers here reflect the degree of distortion when biased gene conversion happens at a given site: the net effect at any given site depends on this and the rate at which the site is subject to gene conversion. The percentage of a markers in a genome associated with gene conversion tracks in any given meiosis varies from $\sim 2 \%$ in yeast to only $\sim 0.005 \%$ in Arabidopsis (Liu et al. 2017), but hotspots can exist. The population genetics of biased gene conversion is similar to that of meiotic drive (Gutz and Leslie 1976), excepting that drive typically happens in every drive heterozygote, while biased gene conversion requires the conversion tract, and heteroduplex, to form around the GC:AT heterozygous site. Just as drive alleles can be deleterious, so too in some cases the favoured allele can be deleterious and such a process could lead to the driven degradation of domains of high recombination.

Importantly, this process of biased transmission provides our best current explanation as to why our genome has blocks of high GC and others with low GC (isochores) and why non-recombining chromosomes and centromeres are AT rich (Eyre-Walker 1993). The high GC domains are crucially domains of high recombination (Duret and Galtier 2009; Fullerton et al. 2001) in which the fixation process, but not the mutation process, is biased towards GC alleles (Duret et al. 2002; Lercher et al. 2002). AT rich regions, by contrast, are low recombination domains in which there is no evidence for a fixation bias and appear to be closer to mutational equilibrium, mutation being GC->AT biased. Thus, the high GC domains are not explained by mutation bias and drift (i.e. not the neutralist model). Possible neutralist models include the notion that the mutation bias varies around the genome, possibly associated with replication time. This model, however, predicts a concordance between the frequency profile of rare and fixed mutations and, assuming compositional equilibrium, that the numbers of GC->AT and AT->GC SNPs should be equal (EyreWalker 1999). However, this concordance isn't seen (Duret et al. 2002; Lercher and Hurst 2002; Lercher et al. 2002).

Selectionist models, that are consistent with this fixation bias, are in principle hard to discriminate from a biased gene conversion model. Such weak selection models suggest that domains of high GC may simply reflect a greater efficiency of selection in domains of high recombination (Charlesworth 1994b) owing the reduced Hill-Robertson interference. A weakness of many such models is that they typically do not specify why, exactly, an AT->GC SNP is selectively favoured, even in non-coding DNA far from gene bodies, they just presume that it could be. In this regard, the model could equally well be consistent with a model supposing that recombination was associated with domains of low GC. Some suggest that increased GC content is an adaptation to higher temperatures (Bernardi and Bernardi 1986), but the overall validity of this is questioned (Eyre-Walker and Hurst 2001), not least because thermophilic prokaryotes aren't GC rich (Galtier and Lobry 
1997; Hurst and Merchant 2001) and isochores aren't unique to homoeotherms (Hughes et al. 1999). Fixation by selection also implies a genetic load associated with their creation and maintenance. It is then curious that isochores are witnessed in organisms with low effective population sizes and low variance in offspring numbers (mammals), where selection is expected to be weakest and load least tolerable, but not seen in fruit flies and yeasts. It is also unclear why the evolution of GC content correlates much more strongly with male recombination than female recombination rates in humans (Duret and Arndt 2008) (see also Popa et al. 2012). It is also questionable as to whether selection can explain the strength of any GC-recombination correlation (Duret and Arndt 2008), although a complicating factor is that, at least in yeast, high GC can predispose to high recombination rates (Kiktev et al. 2018; MarsolierKergoat and Yeramian 2009).

Biased gene conversion, by contrast provides a better specified and more parsimonious model. It requires no increased genetic load, explains a fixation bias, why this bias is associated with domains of high recombination, and importantly, why high recombination is associated with high GC, this being the observed direction of the transmission bias. It also provides a parsimonious explanation as to why GC content variation is declining in some mammals (Duret and Arndt 2008; Duret et al. 2002, 2006). It could potentially explain the sex-specific nature of the GC -recombination correlation (Duret and Arndt 2008) but this has yet to be verified. More generally, one can indeed argue that given the observed gene conversion bias, the evolution of isochores becomes near inevitable so long as recombination is itself concentrated in certain genomic domains. Thus, what was pitched as the ultimate neutralist-selectionist battle (Eyre-Walker and Hurst 2001), is most likely resolved by a third class of explanation: biased transmission (Duret and Galtier 2009).

Is this really a third class of explanation? Some suggest that BGC is a neutral process (Galtier et al. 2001). But the bearers of the advantaged allele can be at a disadvantage and the maths does not resemble drift so much as drive (Gutz and Leslie 1976). It could also be regarded as a form of gamete-level selection, just as meiotic drive can be so configured. However, BGC is different from any mode of selection that requires the allele to be advantageous to the bearer of the allele, be that bearer a haploid gamete or the diploid parent/progeny. It is indeed substantially different from certation in which the allele for which $k>0$ applies is one that makes the pollen bearing it competitively better. Similarly, in cases of meiotic drive where the driving chromosome "kills" the sperm bearing the driven against one (as in $S D$ in Drosophila), one can see the drive allele as giving the sperm bearing it an advantage when in competition against the sperm from the same male not carrying the drive allele. As regards the neutralist-selectionist debate I think it best to consider BGC neither neutralist nor selectionist, and hence that it best considered a third class of model.

It is striking that the literature on BGC and that on selfish elements rarely cross reference each other even though the underlying logic is the same (for an exception see Werren 2011). One reason for this is that unlike meiotic drive genes (SD, $t$-complex, spore killers), there is nothing sophisticated about the allele whose transmission is being affected that renders its transmission distorted: the sophistication is all in the biology of the repair complexes that have evolved the bias.

\section{Is biased gene conversion important in all eukaryotes: a problem with biased technology?}

In many species the extent of the bias in biased gene conversion is strong, with $\sim 70: 30$ bias not being unusual at the site of conversion events. However, is biased gene conversion universal and this strong? The best data derives from Mancera et al.'s array-based study in yeast (Mancera et al. 2008) in which a bias is observed but it is modest at just $50.6 \%$ in favour of GC. We recently tried to replicate this using whole genome sequencing rather than using an array-based method (Liu et al. 2017). We found that if anything the bias is slightly the other way $(50.7 \%$ in favour of AT alleles). It is perhaps striking that the two methods agree to within about $1 \%$. Unfortunately, this is not good enough as the two estimates sit on either side of the key 50:50 threshold. If we merge the two (a sample of over $100,000 \mathrm{AT}<->\mathrm{GC}$ conversion events) there is no significant net bias ( $50.03 \%$ in favour of GC). Making matters worse, owing to the large sample sizes in both studies, the two estimates of bias are significantly different. What is going on? The strains in both instances here were the same, and between strain differences do not seem especially strong (Liu et al. 2017). A possible problem then is a difference in technology: arrays in Mancera et al. versus whole-genome sequencing. Both have recognised biases (see e.g. Benjamini and Speed 2012; Lam et al. 2012; Rieber et al. 2013; Serhal and Lemieux 2013; Uchida et al. 2005; Winchester et al. 2009). SNP arrays for example are thought to be $>99 \%$ accurate (LaFramboise 2009), which may sound impressive but even a $1 \%$ error rate can easily interfere with estimation of a very weak bias. While we validated a subsample of our calls via Sanger sequencing and found no issues, we still do not know the cause of the difference and it is notable that a further recent analysis using the closely related species Saccharomyces paradoxus finds a very weak and barely significant bias in favour of 
GC (Liu et al. 2018). However, as this example shows, when we are looking for miniscule biases then method bias might start to become so prominent that we will need to do more than just sequence more.

\section{Do weak biases matter: the missing concept of the effectively Mendelian allele?}

If the bias is of the order of a tiny deviation from 50:50 would this matter? Put differently, my assertion above that $k$ must be exactly zero might not be correct. In population genetics there is the concept of the effectively neutral allele. This is a Mendelian allele whose effects on fitness are so small that the fixation rates of such alleles are effectively the same as an allele with no effects on fitness, i.e. a strictly neutral allele. Classically, if $s$ is the selective effect of an allele, then if $s \ll 1 / 2 N_{\mathrm{e}}$ it will be effectively neutral, where $N_{\mathrm{e}}$ is the effective population size. In the same context we can consider the effectively Mendelian allele. This would be a neutral allele whose transmission advantage $(k)$ is so small that it behaves as if it were a Mendelian neutral allele. This is a term that, I would suggest, is needed but never employed to the best of my knowledge.

Mathematically, we are not completely in the dark about such a concept. Consideration of biased gene conversion has noted the mathematical resemblance to positive selection (Nagylaki 1983) and extension of diffusion models have permitted estimation of equilibrium GC content under additive assumptions incorporating biased gene conversion (Marais et al. 2004). This direct correspondence between biased gene conversion and weak positive selection is in many regards bad news for the technology bias. A bias as small as 50.03:49.97 could in a selectionist framework be considered an allele under strong selection, so long as the effective population size is great enough and gene conversion acts on the site regularly enough. For a regularly sexual species with effective population sizes in the millions, a utterly miniscule net bias from 50:50 could behave as if non-neutral. Good luck trying to detect that!

\section{Discussion}

While absence of bias in segregation ratios was key to the understanding that selection as a force can matter, a century later focus has to some degree shifted to the possibility that biased segregation matters, especially for processes like biased gene conversion. As I have argued, however, resolving subtle but biologically important biases puts us ever closer to the limits of technology, owing to their intrinsic biases. The centrality of transmission bias (or lack thereof) to understanding the fate of alleles also underpins the more general notion that to understand evolution, we need to understand the underlying transmission genetics, which in turn requires us to understand molecular genetics (for example the molecular biology of gene conversion).

To some extent the last century was one in which understanding of transmission and population genetics predominated in the pre-DNA era, while molecular biology was immensely successful in the post DNA era. But, I would suggest, while there certainly has been interaction between molecular and population genetics, not least through population and evolutionary genetical analysis of sequence data, they have not had as much to say to each other as they might have, perhaps owing to the usual ghettoization of academic disciplines. Evolutionary biologists have much to learn from really understanding the black box of molecular genetics. The example of biased gene conversion is a case in point. This would be an example where the assumption that one can just study phenotype and ignore the underlying genetics - the phenotypic gambit (Grafen 2014) - fails (see also Hadfield et al. 2007)). Equally, those that study the basics of molecular biology and molecular genetics can similarly enrich their understanding through appreciation of the nuances of population and evolutionary genetics. For example, acknowledging that species with small populations are expected to have bloated error-prone genomes (Lynch and Conery 2003), simply because selection is inefficient when $N_{\mathrm{e}}$ is low, can add a necessary caution to a potentially futile search for a function for all things. Now more than ever these distinct disciplines could benefit from sitting more closely under one umbrella, with biases about the value of one subject area over the other left to the side.

\section{Bias and pedagogy}

What in broader scope are we to make of this history? To those of us evolutionary biologists wedded to genetics, it seems quite trivial that you cannot fully understand evolution without an understanding of genetics. And yet, in UK schools the two subjects are taught as distinct entities with no connection (selection alone matters and the phenotypic gambit is implicitly made). So surprised was I to discover this that we set up a large scale randomised control trial to examine the role of teaching order in secondary schools (Mead et al. 2017). Some classes were taught evolution then genetics, the others genetics then evolution. Teaching genetics before evolution led to no diminution of genetics understanding (compared to the other way around) but improved evolution understanding 5-10\% compared with teaching in the opposite order (Mead et al. 2017).

One hypothesis to explain why teaching genetics first is so successful is that a grounding in genetics renders 
evolution a logical necessity, so making it easier to understand as a process: if you understanding DNA, you can understand mutations and hence alleles, if you understand alleles you understand that they can change frequency and hence that populations can genetically change over time. If so, then reinforcing the multiple different reasons that alleles might change frequency (neutral evolution, classical selection, biased transmission) should help solidify the connection between DNA, mutation and evolution and in turn, the inevitability of genetical change of populations. Teaching the importance of biased and unbiased transmission may then be an effective means to breakdown psychological biases that cause cognitive dissonance so impeding learning about evolution (Lawson and Worsnop 1992; McKeachie et al. 2002), although the importance of this may vary between the US and the UK (Mead et al. 2018). This remains to be tested, but we know that when students learn effectively about evolution their acceptance of evolution (different from their understanding of evolution) also goes up (Mead et al. 2017), suggesting, optimistically, that better understanding has some role in dissolving bias.

Acknowledgements I would like to acknowledge invaluable comments from Barbara Mable, Alison Woollard, Jonathan Pettitt and Brian Charlesworth. L.D.H. is funded by European Research Council grant ERC-2014-ADG 669207.

\section{Compliance with ethical standards}

Conflict of interest The author declares that he has no conflict of interest.

Publisher's note: Springer Nature remains neutral with regard to jurisdictional claims in published maps and institutional affiliations.

\section{References}

Agren JA, Clark AG (2018) Selfish genetic elements. PLoS Genet 14: e1007700

Bengtsson BO (1985) Biased conversion as the primary function of recombination. Genet Res 47:77-80

Benjamini Y, Speed TP (2012) Summarizing and correcting the GC content bias in high-throughput sequencing. Nucleic Acids Res 40:e72

Bernardi G, Bernardi G (1986) Compositional constraints and genome evolution. J Mol Evol 24:1-11

Boveri T (1904) Ergebnisse über die Konstitution der chromatischen Substanz des Zellkerns. G. Fischer, Jena

Brown TC, Jiricny J (1988) Different base base mispairs are corrected with different efficiencies and specificities in monkey kidneycells. Cell 54:705-711

Bruck D (1957) Male segregation ratio as a factor in maintaining lethal alleles in wild populations of house mice. Proc Natl Acad Sci USA 43:152-158

Bulmer M (2004) Did Jenkin's swamping argument invalidate Darwin's theory of natural selection? Br J Hist Sci 37:281-297
Burt A, Trivers R (2008). Genes in conflict: the biology of selfish genetic elements. Belknap, Cambridge

Carothers EE (1913) The Mendelian ratio in relation to certain orthopteran chromosomes. J Morphol 24:487-511

Castle WE, Little CC (1910) On a modified Mendelian ratio among yellow mice. Science 32:868-870

Cavalier-Smith T (1980) How selfish is DNA? Nature 285:617-618

Charlesworth B (1994a) The evolution of lethals in the t-haplotype system of the mouse. Proc R Soc Lond Ser B 258:101-107

Charlesworth B (1994b) Patterns in the genome. Curr Biol 4:182-184

Correns C (1902) Scheinbare Ausneh men von der Mende1s 'schen Spaltungs regel fUr Bastarde. Ber Dtsch Bot Gesells 20:159

Cosmides LM, Tooby J (1981) Cytoplasmic inheritance and intragenomic conflict. J Theor Biol 89:83-129

Crow EW, Crow JF (2002) 100 years ago: Walter Sutton and the chromosome theory of heredity. Genetics 160:1-4

Crow JF (1991) Why is Mendelian segregation so exact? Bioessays 13:305-312

Darlington CD, Thomas P (1941) Morbid mitosis and the activity of inert chromosomes in Sorghum. Proc R Soc, Lond, Ser B 130:127-150

Darwin CR (1868) The variation of animals and plants under domestication, vol. 2. John Murray, London

Davis AS (1871) The north British review and the origin of species. Nature 5:161

Dunn LC (1953) Variations in the segregation ratio as causes of variations in gene frequency. Acta Genet Stat Med 4:139-147

Duret L, Arndt PF (2008) The impact of recombination on nucleotide substitutions in the human genome. PLoS Genet 4:e1000071

Duret L, Eyre-Walker A, Galtier N (2006) A new perspective on isochore evolution. Gene 385:71-74

Duret L, Galtier N (2009) Biased gene conversion and the evolution of mammalian genomic landscapes. Annu Rev Genom Hum Genet 10:285-311

Duret L, Semon M, Piganeau G, Mouchiroud D, Galtier N (2002) Vanishing GC-rich isochores in mammalian genomes. Genetics 162:1837-1847

Eshel I (1985) Evolutionary genetic stability of Mendelian segregation and the role of free recombination in the chromosomal system. Am Nat 125:412-420

Eyre-Walker A (1993) Recombination and mammalian genome evolution. Proc R Soc Lond Ser B 252:237-243

Eyre-Walker A (1999) Evidence of selection on silent site base composition in mammals: Potential implications for the evolution of isochores and junk DNA. Genetics 152:675-683

Eyre-Walker A, Hurst LD (2001) The evolution of isochores. Nat Rev Genet 2:549-555

Federley H (1911). Sur un cas d'heredite gynephore dans une espece de papillon. IV Conf. Int. de Genetique, Paris, pp 469-477

Federley H (1936) Sex limited heredity cancer in Lepidopters larvae. Hered Lund 22:193-216

Feldman MW, Otto SP (1991) A comparative approach to the theoretical population-genetics theory of segregation distortion. Am Nat 137:443-456

Festinger L (1957) A Theory of cognitive dissonance. Stanford University Press, Stanford, CA

Frank SA (1991) Divergence of meiotic drive-suppression systems as an explanation for sex-biased hybrid sterility and inviability. Evolution 45:262-267

Fullerton SM, Bernardo Carvalho A, Clark AG (2001) Local rates of recombination are positively correlated with GC content in the human genome. Mol Biol Evol 18:1139-1142

Galtier N, Lobry JR (1997) Relationships between genomic G+C content, RNA secondary structures, and optimal growth temperature in prokaryotes. J Mol Evol 44:632-636 
Galtier N, Piganeau G, Mouchiroud D, Duret L (2001) GC-content evolution in mammalian genomes: The biased gene conversion hypothesis. Genetics 159:907-911

Gershenson S (1928) A new sex ratio abnormality in Drosophila obscura. Genetics 13:488-507

Grafen A (2014) The formal darwinism project in outline. Biol Philos 29:155-174

Gutz H, Leslie JF (1976) Gene conversion: a hitherto overlooked parameter in population genetics. Genetics 83:861-866

Hadfield JD, Nutall A, Osorio D, Owens IP (2007) Testing the phenotypic gambit: phenotypic, genetic and environmental correlations of colour. J Evol Biol 20:549-557

Hagemann R (2000) Erwin Baur or Carl Correns: Who really created the theory of plastid inheritance? J Hered 91:435-440

Haldane JBS (1932) The causes of evolution. Cornell University Press, New York

Halldorsson BV, Hardarson MT, Kehr B, Styrkarsdottir U, Gylfason A, Thorleifsson $\mathrm{G}$ et al. (2016) The rate of meiotic gene conversion varies by sex and age. Nat Genet 48:1377-1384

Hamilton WD (1967) Extraordinary sex ratios. Science 156:477-488

Hardy GH (1908) Mendelian proportions in a mixed population. Science 28:49-50

Hauschteck-Jungen E (1990) Postmating reproductive isolation and modification of the "sex rati" trait in Drosophila subobscura induced by the sex chromosome gene arrangement $\mathrm{A}_{2+3+5+7}$. Genetica 83:31-44

Heribert-Nilsson N (1920) Zuwachsgeschwindigkeit der pollenschlauche und gestorte Mendeszahlen bei Oenothera lamarkiana. Hereditas 1:41-67

Heribert-Nilsson N (1923) Zertationsversuche mit durchtrennung des griffels bei Oenothera lamarckiana. Hereditas 4:177-190

Hiraizumi Y, Sandler L, Crow JE (1960) Meiotic drive in naturalpopulations of Drosophila melanogaster. 3. Populational implications of the segregation-distorter locus. Evolution 14:433-444

Hughes S, Zelus D, Mouchiroud D (1999) Warm-blooded isochore structure in Nile crocodile and turtle. Mol Biol Evol 16:1521-1527

Hurst GDD, Schilthuizen M (1998) Selfish genetic elements and speciation. Heredity 80:2-8

Hurst LD, Atlan A, Bengtsson BO (1996) Genetic conflicts. Q Rev Biol 71:317-364

Hurst LD, Merchant AR (2001) High guanine-cytosine content is not an adaptation to high temperature: a comparative analysis amongst prokaryotes. Proc R Soc Lond B 268:493-497

Hurst LD, Pomiankowski A (1991) Causes of sex ratio bias may account for unisexual sterility in hybrids: a new explanation of Haldane's rule and related phenomena. Genetics 128:841-858

Jenkin F (1867) Review of the origin of species. North Br Rev 46:277-318

Kiktev DA, Sheng Z, Lobachev KS, Petes TD (2018) GC content elevates mutation and recombination rates in the yeast Saccharomyces cerevisiae. Proc Natl Acad Sci USA 115:E7109-E7118

LaFramboise T (2009) Single nucleotide polymorphism arrays: a decade of biological, computational and technological advances. Nucleic Acids Res 37:4181-4193

Lam HYK, Clark MJ, Chen R, Chen R, Natsoulis G, O'Huallachain M et al. (2012) Performance comparison of whole-genome sequencing platforms. Nat Biotechnol 30:78-U118

Lamb BC (1984) The properties of meiotic gene conversion important in its effects on evolution. Heredity 53:113-138

Lamb BC (1985) The effects of mispair and nonpair correction in hybrid DNA on base ratios $(\mathrm{G}+\mathrm{C}$ content $)$ and total amounts of DNA. Mol Biol Evol 2:175-188

Lane N (2014) Bioenergetic constraints on the evolution of complex life. Cold Spring Harb Perspect Biol 6:a015982
Lane N, Martin W (2010) The energetics of genome complexity. Nature 467:929-934

Lane N, Martin WF (2016) Mitochondria, complexity, and evolutionary deficit spending. Proc Natl Acad Sci USA 113:E666

Lawson AE, Worsnop WA (1992) Learning about evolution and rejecting a belief in special creation: effects of reflective reasoning skill, prior knowledge, prior belief and religious commitment. J Res Sci Teach 29:143-166

Leigh EG (1971) Adaptation and diversity. Freeman, Cooper and Co., San Francisco

Leigh Jr EG (1990) Afterword to the causes of evoluion by J.B.S Haldane. Princeton University Press, Princeton, NJ

Lercher MJ, Hurst LD (2002) Can mutation or fixation biases explain the allele frequency distribution of human single nucleotide polymorphisms (SNPs)? Gene 300:53-58

Lercher MJ, Smith NG, Eyre-Walker A, Hurst LD (2002) The evolution of isochores. evidence from SNP frequency distributions. Genetics 162:1805-1810

Lesecque Y, Mouchiroud D, Duret L (2013) GC-biased gene conversion in yeast is specifically associated with crossovers: molecular mechanisms and evolutionary significance. Mol Biol Evol 30:1409-1419

Lewis D (1941) Male sterility in natural populations of hermaphroditic plants. New Phytol 40:158-160

Liu H, Huang J, Sun X, Li J, Hu Y, Yu L et al (2017) Tetrad analysis in plants and fungi finds large differences in gene conversion rates but no GC bias. Nat Ecol Evol 2:164-173

Liu H, Maclean CJ, Zhang J (2018) Evolution of the yeast recombination landscape. Mol Biol Evol 36:412-422

Lynch M, Conery JS (2003) The origins of genome complexity. Science 302:1401-1404

Lynch M, Marinov GK (2016) Mitochondria do not boost the bioenergetic capacity of eukaryotic cells. Proc Natl Acad Sci USA 113:E667-E668

Lynch M, Marinov GK (2017) Membranes, energetics, and evolution across the prokaryote-eukaryote divide. Elife 6:e20437

Lynch M, Marinov GK (2018) Response to Martin and colleagues: mitochondria do not boost the bioenergetic capacity of eukaryotic cells. Biol Direct 13:26

Lyon MF (1992) Deletion of mouse t-complex distorter-1 produces an effect like that of the t-form of the distorter. Genet Res 59:27-33

Lyon MF (2003) Transmission ratio distortion in mice. Annu Rev Genet 37:393-408

Lyttle TW (1991) Segregation distorters. Annu Rev Genet 25:511-557

Mancera E, Bourgon R, Brozzi A, Huber W, Steinmetz LM (2008) High-resolution mapping of meiotic crossovers and noncrossovers in yeast. Nature 454:479-485

Marais G (2003) Biased gene conversion: implications for genome and sex evolution. Trends Genet 19:330-338

Marais G, Charlesworth B, Wright SI (2004) Recombination and base composition: the case of the highly self-fertilizing plant Arabidopsis thaliana. Genome Biol 5:art. no.-R45

Marsolier-Kergoat MC, Yeramian E (2009) GC content and recombination: reassessing the causal effects for the Saccharomyces cerevisiae genome. Genetics 183:31-38

McDermott SR, Noor MAF (2010) The role of meiotic drive in hybrid male sterility. Philos Trans R Soc B 365:1265-1272

McKeachie WJ, Lin Y-G, Strayer J (2002) Creationist vs. evolutionary beliefs: effects on learning biology. Am Biol Teach 64:189-192

Mead R, Hejmadi M, Hurst LD (2017) Teaching genetics prior to teaching evolution improves evolution understanding but not acceptance. PLoS Biol 15:e2002255

Mead R, Hejmadi M, Hurst LD (2018) Scientific aptitude better explains poor responses to teaching of evolution than psychological conflicts. Nat Ecol Evol 2:388 
Metz CW, Moses MS (1923) Chromosomes in Drosophila. J Hered 14:195-204

Morgan TH, Bridges CB, Sturtevant AH (1925) The genetics of Drosophila. Bibliogr Genet 2:1-262

Morris SW (1994) Fleeming Jenkin and the origin of species: a reassessment. Br J Hist Sci 27:313-343

Nagylaki T (1983) Evolution of a large population under gene conversion. Proc Natl Acad Sci USA 80:5941-5945

Östergren G (1945) Parasitic nature of extra fragment chromosomes. Bot Not 2:157-163

Patten MM (2018) Selfish X chromosomes and speciation. Mol Ecol 27:3772-3782

Phadnis N, Orr HA (2009) A single gene causes both male sterility and segregation distortion in Drosophila hybrids. Science 323:376-379

Popa A, Samollow P, Gautier C, Mouchiroud D (2012) The sexspecific impact of meiotic recombination on nucleotide composition. Genome Biol Evol 4:412-422

Presgraves DC (2010) The molecular evolutionary basis of species formation. Nat Rev Genet 11:175-180

Prout T (1953) Some effects of variations in the segregation ratio and of selection on the frequency of alleles under random mating. Acta Genet Stat Med 4:148-151

Rieber N, Zapatka M, Lasitschka B, Jones D, Northcott P, Hutter B et al. (2013) Coverage bias and sensitivity of variant calling for four whole-genome sequencing technologies. PLoS ONE 8: e66621

Robinson MC, Stone EA, Singh ND (2014) Population genomic analysis reveals no evidence for GC-biased gene conversion in Drosophila melanogaster. Mol Biol Evol 31:425-433

Sandler L, Novitski E (1957) Meiotic drive as an evolutionary force. Am Nat 41:105-110

Serhal P, Lemieux S (2013) Correction of spatial bias in oligonucleotide ArrayData. Adv Bioinforma 2013:167915

Simmonds HW (1923a). All female families of Hypolimnas bolina L. bred in Fiji by H.W. Simmonds. Proc Ent Soc 1923: ix-xii

Simmonds HW (1923b) Families of Hypolimnas bolina L., bred from known female parents from different Fijian islands. Trans Ent Soc Lond 1923:651-662

Simmonds HW (1926) Sex ratio of Hypolimnas bolina L. in Viti Levu, Fiji. Proc Ent Soc 1:29-31
Simmonds HW (1928) Mr. Simmonds' conclusion that all-femaleproducing females form a persistent strain in Suva, Fiji. Proc Ent Soc 3:43-44

Smeds L, Mugal CF, Qvarnstrom A, Ellegren H (2016) Highresolution mapping of crossover and non-crossover recombination events by whole-genome re-sequencing of an avian pedigree. PLoS Genet 12:e1006044

Sturtevant AH (1926) Renner's studies on the genetics of Oenothera. Q Rev Biol 1:283-288

Sutton W (1902) On the morphology of the chromosome group in Brachystola magna. Biol Bull 4:24-39

Sutton W (1903) The chromosomes in heredity. Biol Bull 4:231-251

Tao Y, Hartl DL, Laurie CC (2001) Sex-ratio segregation distortion associated with reproductive isolation in Drosophila. Proc Natl Acad Sci USA 98:13183-13188

Uchida S, Nishida Y, Satou K, Muta S, Tashiro K, Kuhara S (2005) Detection and normalization of biases present in spotted cDNA microarray data: A composite method addressing dye, intensitydependent, spatially-dependent, and print-order biases. DNA Res $12: 1-7$

van der Gaag M, Debets AJM, Oosterhof J, Slakhorst M, Thijssen JAGM, Hoekstra RF (2000) Spore-killing meiotic drive factors in a natural population of the fungus Podospora anserina. Genetics 156:593-605

Wallberg A, Glemin S, Webster MT (2015) Extreme recombination frequencies shape genome variation and evolution in the honeybee, Apis mellifera. PLoS Genet 11:e1005189

Weinberg W (1908) Über den Nachweis der Vererbung beim Menschen. Jahresh Des Ver für Vaterl Nat Württemberg 64:368-382

Werren JH (1987) The coevolution of autosomal and cytoplasmic sexratio factors. J Theor Biol 124:317-334

Werren JH (2011) Selfish genetic elements, genetic conflict, and evolutionary innovation. Proc Natl Acad Sci USA 108:10863-10870

Werren JH, Nur U, Wu CI (1988) Selfish genetic elements. Trends Ecol Evol 3:297-302

Williams AL, Genovese G, Dyer T, Altemose N, Truax K, Jun G et al (2015) Non-crossover gene conversions show strong GC bias and unexpected clustering in humans. Elife 4:e04637

Winchester L, Yau C, Ragoussis J (2009) Comparing CNV detection methods for SNP arrays. Brief Funct Genom 8:353-366 